\title{
The effect of age on visuomotor learning processes
}

\author{
Chad Michael Vachon ${ }^{1,3}$, Shanaathanan Modchalingam ${ }^{1,2}$, Bernard Marius 't Hart' ${ }^{* 1}$, \\ Denise Y. P. Henriques ${ }^{1-3}$
}

${ }^{1}$ Centre for Vision Research, York University, Toronto, Ontario, Canada

${ }^{2}$ School of Kinesiology and Health Science, York University, Toronto, Ontario, Canada

${ }^{3}$ Department of Psychology, York University, Toronto, Ontario, Canada

${ }^{*}$ Corresponding author: thartbm@gmail.com

\section{Abstract}

People can rapidly adapt their movements to a wide array of changes in our environment or the motor apparatus, although this ability can decline with age. Adaptation of movements, such as reaching with altered visual feedback, is thought to rely on the quality of sensory feedback and how well we can predict our movements. Cognitive strategies can also contribute to how quickly adaptation can occur. Agerelated declines in sensory acuity and cognitive function, such as strategy use, may explain poorer adaptation in older adults compared to younger adults. The current study tested the effects of instruction and strategy use on how well older $(n=38)$ and younger $(n=42)$ adults were able to compensate for a $30^{\circ}$ visuomotor rotation during reachtraining and use this strategy afterwards when reaching without a cursor. Next, traininginduced changes in proprioceptive and predicted estimates of the adapted hand in these two age groups were compared. This was done by having older and younger adults estimate the location of their unseen hand when it was either moved out by a robot (passive localization: proprioception only, no prediction) or was moved by the participant themselves (active localization: prediction and proprioception) before and after visuomotor adaptation. Any difference between these localization tasks shows changes in predicted or efferent-based estimates. Instruction benefitted older adults less than younger adults during initial reach training, but a similar pattern in reach aftereffects in the two age groups suggests that older adults' strategy use could be evoked during no-cursor reaches after enough training. Following visuomotor adaptation, older adults, whether instructed or not, showed slightly larger visually driven changes in their passive or proprioceptive hand estimates but not their efferent-based or predicted estimates of hand position. These results suggest that older adults do not differ much from younger adults in their ability to adapt their reaching movements implicitly or with the use of cognitive strategies; however their hand estimates based on updated proprioception are more affected by visual training. (data: https://osf.io/qzhmy) 


\section{Introduction}

Our brain has evolved to adapt our movements to persistent changes in our environment and in our body. However, our ability to adapt reaching movements appears to diminish with age. Reach adaptation is based on both explicit and implicit processes and these are thought to be affected by aging, but it is unclear how. The goal of this study is to understand how aging contributes to changes in these processes.

Older adults can adapt their reaches to altered visual feedback of the hand, but in some cases have slower or incomplete learning compared to younger adults. Specifically, when adapting to larger cursor rotations (such as $60^{\circ}$ or $90^{\circ}$ ), older adults do not adapt at the same rate nor to the same extent as younger adults [1-7]. A similar difference between age-groups can be seen in force-field adaptation [8,9] as well as prism adaptation [10] although not for [11]. However, when the visual perturbation is small (such as $30^{\circ}$ ), age-related differences in initial stages of motor learning are less robust: with some studies showing a deficit for older adults [12] and others not [4,7,11]. Adaptation may include both explicit and implicit learning, while reach aftereffects, the continued change in performance even in the absence of the perturbation, are considered to reflect only an implicit component of learning. Older adults tend to produce similar or even larger reach aftereffects than younger adults [1-4,7,10,13-15], suggesting that implicit learning may be preserved. The age-related decline in the capacity to adapt movements under certain contexts and not others suggests that agerelated differences in motor learning might be largely due to changes in explicit processes rather than implicit processes. However, to what extent age influences explicit and implicit components of visuomotor learning is largely unknown. Therefore, in this study, we tested whether aging affects our ability to initially make use of a cognitive strategy during learning as well as the resulting implicit changes in state estimates and motor learning.

Adaptation requires both a cognitive, or explicit, component which tends to contribute to early stages of learning, as well as implicit processes that predominate in the later stages. The explicit component has been assessed by having participants indicate their aiming strategy either in a trial-by-trial basis $[4,16]$, or indicate their developed strategy post-adaptation when reaching without a perturbation [4,5,17-20]. In addition, explicit instruction about how to compensate for a visual perturbation have been used to facilitate initial learning [16,19-21]. Some other evidence for the role of explicit processes for motor learning originates from research that shows that in some cases, a simultaneously performed secondary task can affect performance during training or post-training performance during force field adaptation [22-24] although no such clear effect has been shown for visuomotor adaptation, for example simultaneous rapid serial response tasks do not affect learning rate $[25,26]$. Because larger rotations produce larger initial reaching errors than smaller rotations, they are more likely to require and evoke cognitive processes, such as those associated with strategy use to compensate for a rotation. This also explains why the explicit processes emerge early in training when the reaching errors from the perturbation are largest and easiest to detect [16]. 
This is in contrast with smaller or gradually-introduced perturbations, where reaching errors are smaller and less salient, and therefore may exclusively reflect implicit processes. The resulting reach aftereffects that follow adaptation to all sizes of perturbations reflect these implicit motor and possibly sensory changes. Thus, explicit and implicit processes appear to contribute to different aspects of adaptation performances.

A possible explanation for age-related differences in initial learning for larger perturbations; less consistent differences for smaller perturbations; and similar reach aftereffects in all cases, may be found in differences in how explicit and implicit processes change with age. This hypothesis is somewhat supported by the work of $[14,27,28]$. Using a verbal aiming-strategy report before and after training with a $75^{\circ}$ rotated cursor, they found that older adults used their strategy less, as they underestimated the angles needed to reach to the target with the perturbation. However, this group did not find this effect with a $30^{\circ}$ rotated cursor. Some studies suggest that for younger adults, lower spatial working memory or performance on a secondary memory task is correlated with poorer learning rates $[1,22,29]$. Thus, while it hasn't been demonstrated in older adults [2], it could be inferred [7] that age-related cognitive decline $[30,31]$ can also explain age-related differences in adaptation rates. In this study, we will assess the extent that aging affects the ability to initially make use of or develop explicit strategies during visuomotor adaptation.

Adaptation leads not only to implicit motor changes that persist even when the perturbation is removed, but also changes in proprioceptive estimates of hand location. Proprioceptive estimates of hand position have been robustly shown to shift, or be recalibrated, following both visuomotor adaptation [15,32-38] and force field adaptation $[39,40]$. In the case of visuomotor adaptation, estimates of the unseen hand's location shift in the direction of the visual representation of the hand during training, usually by $\sim 20 \%$ of the visuomotor distortion $[15,32-37,41]$. In turn, this visually-induced change in hand proprioception appears to contribute to changes in motor performance, particularly reach aftereffects $[13,42-45]$. These changes in proprioception seem to be largely implicit, in that they are not affected by instructions [18], are similar following both abrupt and gradual introduction to the perturbation [46], and do not change with age when using the usual perceptual reports $[15,44]$. Thus, if the implicit contributions to learning remain largely intact across age, we predict that, like reach aftereffects, proprioceptive recalibration should not differ between older and younger adults.

Like proprioception, efferent-based hand position estimates are an important contributor to state estimation and are central to adaptation. In fact, many computational models of motor learning specify that the forward model, used to generate predicted sensory consequences based on efferent signals, must be updated for implicit motor learning to occur [47]). The amount of change in these state estimates has been quantified by measuring changes in hand localization following visuomotor adaptation $[18,33,35,48,49]$. Synofzik et al. [48] and Izawa et al. [49] found that cerebellar patients show significant but smaller learning-induced changes in hand estimates, compared to healthy controls and concluded that the reduction reflects the critical role that the 
cerebellum plays in updating predicted sensory consequences for learning. Further, the persistent shifts in hand localization in these patients suggest that perhaps the part of these learning-induced changes that may be due to proprioceptive recalibration could occur outside the cerebellum [44]. The contributions of the afferent and efferent sources of these changes in hand localization have been recently investigated in young adults $[18,33,35,50]$. However, it is not clear how predicted estimates of hand location change with healthy aging. Nonetheless, given that efferent-driven forward models contribute to implicit changes in sensory and motor performance, we do not expect age-related declines in either proprioceptive or predictive changes in hand localization estimates following adaptation.

Here we investigate the extent by which age-related deficits in motor learning could be explained by the distinct effects of age on explicit and implicit processes. First, we test if there are any age-related differences in cognitive strategies during and after motor adaptation by assessing the impact of instruction. Then, we assess whether there are age-related differences in people's ability to evoke a strategy when reaching without a cursor, independent of whether they were instructed or not. Finally, we test whether aging affects implicit motor and proprioceptive changes, as well as updates in predicted sensory consequences of hand location estimates. Our results provide a more comprehensive understanding of how age affects the contributions of explicit and implicit processes in motor learning.

\section{Methods}

\section{Participants}

Forty-one younger (mean age of 20.9 years, SD $=2.77$ ) and thirty-eight older (mean age of 70.0 years, $S D=6.78$ ) adults were recruited through various research participant pools at York University: the Undergraduate Research Participant Pool (URPP), Kinesiology Undergraduate Research Participant Pool (KURE), York Research Participant Pool (YRPP), and also from the surrounding community. The participants from the undergraduate research pools were given course credit for participation. Older adults who were recruited through the YRPP or the community were paid a honorarium and lunch, as required by the YRPP, to compensate for time and travel to the university. All participants had normal or corrected-to-normal visual acuity, were right handed, and self-reported that they were in good health and were able to understand the tasks. Participants gave their informed written consent before taking part in the study. The York Human Participants Review Sub-committee approved this study (\#2014-240).

All participants adapted to a $30^{\circ}$ visuomotor rotation, however after the first session of baseline training, half of each age group were given explicit instructions on the nature of the perturbation, and a strategy to counter the rotation, while the other half received no instructions. Thus, there were four groups: non-instructed younger adults ( $n=20,14$ female), instructed younger adults $(n=21,13$ female), non-instructed older adults $(n=$ 19, 6 female) and instructed older adults ( $n=19,14$ female). The hand path and velocity profile of every single reach trial (with cursor or without) and the endpoint for 
every localization trial were visually inspected for quality to remove trials with obvious measurement or task errors, such as failure to reach the target, understand task instructions, or localization responses that did not land near the visually presented arc as required. The participant numbers mentioned above, do not include 4 younger and 3 older participants who failed to complete the tasks as instructed.

\section{Setup}

Participants were seated in front of a table on a height-adjustable chair so that they could comfortably see and reach to displayed targets projected on a reflective surface from a monitor (Samsung $510 \mathrm{~N}, 60 \mathrm{~Hz}$ ), located $28 \mathrm{~cm}$ above a 2-joint robot manipulandum (Interactive Motion Technologies Inc., Cambridge, MA, USA). The reflective surface was $14 \mathrm{~cm}$ above the robot manipulandum and $14 \mathrm{~cm}$ below the downward facing monitor (Figure 1A). The chair was fixed in its position for the duration
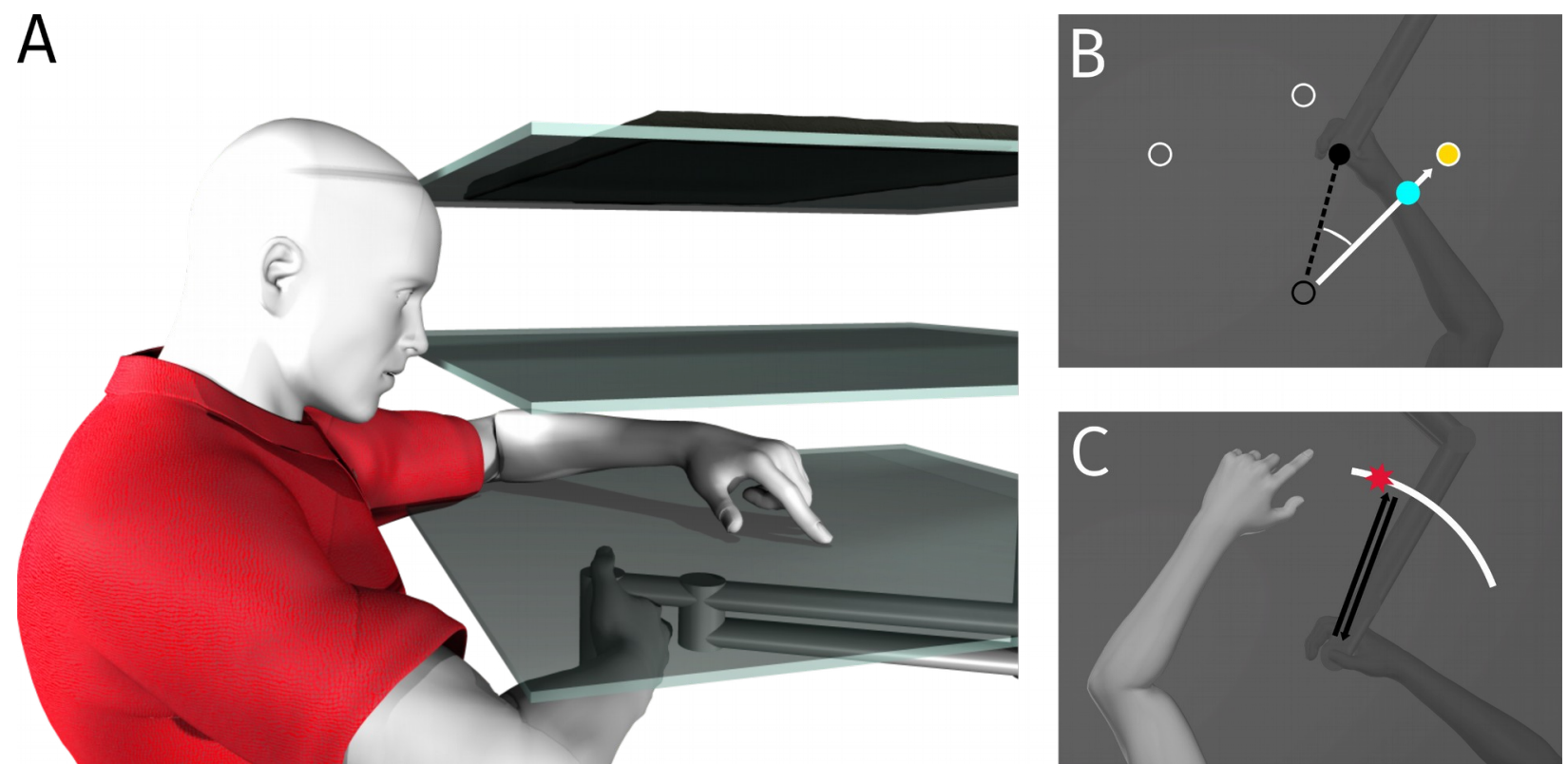

Figure 1. Apparatus and experimental setup. A: Participants moved their right hand while gripping the handle of a two-joint robot manipulandum. Stimuli are produced by a downward-facing monitor (top surface) above a reflective surface (middle). The touchscreen panel is located below the reflective surface and just above the robot handle (bottom). B: Training task: The three white hollow circles represent the targets. They can either be directly in front of the home position, $45^{\circ}$ clockwise (CW) or counter clockwise (CCW) from it. During the main rotated reach training, the cursor was blue and rotated $30^{\circ} \mathrm{CW}$ (white solid vector) relative to the actual (unseen) hand position/direction (black dashed vector). C: Localization task: Using 't Hart and Henriques [33] localization task, the unseen right hand was moved to a location along a white arc either by participants voluntarily moving their hand or having their hand physically moved by the robot. Once participants returned their right hand to the home position, they indicated on the touchscreen with their visible left hand, where their trained right hand had intersected the arc (illustrated by red star). 
of the experiment. Participants were asked to grip a vertical handle with their right hand. They were instructed to place their right thumb on a screw located on top of the robot handle. The handle was attached to the free end of the robot manipulandum and could be moved on a horizontal plane. A thick black cloth was draped and tucked over participants' right shoulder to ensure that they did not see their right arm.

Since the reflective surface was located halfway between the monitor and the robot manipulandum, the reflection of the cursor and the targets appeared on the same plane as the thumb of the right hand. The reflective surface also occluded the right hand and arm.

During some tasks, participants used their visible left hand, which was then illuminated by a lamp, to respond on a touchscreen panel (Keytec Inc., Garland, TX, USA sampled at a resolution of $1440 \times 900$ pixels) located $2 \mathrm{~cm}$ above the robot handle, to indicate the perceived position of their unseen right thumb (Figure $1 \mathrm{~A}$ and $\mathrm{C}$ ).

\section{Procedure}

The procedure and set up are similar to our previous study [18]. It includes two experimental sessions (Figure 2), and each session had several repetitions of the same four tasks (described in detail below) The first session (illustrated on the top row of Figure 2) measured baseline results where the cursor was aligned with the hand during reach training. The second session (bottom row) had training with a $30^{\circ} \mathrm{CW}$ rotated cursor as illustrated in Fig $1 \mathrm{~b}$. Initial reach training was followed by several other tasks (see Figure 2) to measure training-induced changes, interleaved with additional training to counter decay. Each block of tasks was repeated four times in each session. During the break between the two sessions, half of the participants were instructed about the nature of this $30^{\circ}$ perturbation in the second session while the other half were not. Some older participants took additional breaks, as desired, but only right before a reach

training task. After completing both sessions of the experiment, participants were then asked a series of questions to assess awareness of the perturbation (https://osf.io/qzhmy/).

Before each task started, a short reminder of what to do was displayed. Each task consisted of a number of trials, and involved one of several kinds of hand displacements. At the beginning of each trial the hand was locked into place for $300 \mathrm{~ms}$ at the home position $(\sim 20 \mathrm{~cm}$ in front of the participant on their body midline). While the hand was locked in place, either a target or an arc was displayed. After the hand displacement trial, participants returned their hand to the home position along a constrained straight path, generated by a perpendicular resistant force of $2 \mathrm{~N} /(\mathrm{mm} / \mathrm{s}$ ) and a viscous damping of $5 \mathrm{~N} /(\mathrm{mm} / \mathrm{s})$, to begin the next trial. The different tasks are described below.

Training. During reach training tasks (Figure 1B), participants' hand position was represented as a cursor $(1.0 \mathrm{~cm}$ in diameter) which was green when it moved in alignment with the hand, and blue when it represented the hand position rotated $30^{\circ}$ 
around the home position. At the beginning of the trial the hand was locked into place, while a target was displayed as a yellow dot $(1.0 \mathrm{~cm}$ in diameter) located $12 \mathrm{~cm}$ straight in front of the home position $\left(\right.$ at $\left.90^{\circ}\right)$ or diagonally to the right $\left(45^{\circ}\right)$ or to the left $\left(135^{\circ}\right)$. After the hand was released, the participant was to move the cursor to the target, i.e. the center of the hand cursor was within $0.5 \mathrm{~cm}$ of the target's center. Then the target and cursor disappeared and participants moved their hand back to the home position along a constrained straight path, to begin the next trial.

In the first session, (top row of Figure 2), the cursor was aligned with the hand position. The first aligned training task included 45 trials, in between the other tasks (localization and no-cursor tasks), additional blocks of 9 identical training trials were performed. In the second session (bottom row of Figure 2), the cursor represented the hand position, but rotated by $30^{\circ} \mathrm{CW}$ relative to the home position. The first rotated training task included 90 trials while subsequent rotated training tasks consisted of 30 trials to keep adaptation saturated.

Instructions. For both the older and younger adult groups, half of the participants were instructed on a strategy to counteract the $30^{\circ} \mathrm{CW}$ cursor rotation, and the other half did not get this instruction. Instruction was provided using an animation as well as a verbal explanation and a clock diagram (used in [21]), where 1 hour represents $30^{\circ}$ (see https://osf.io/qzhmy/). It was ensured that participants understood these instructions;

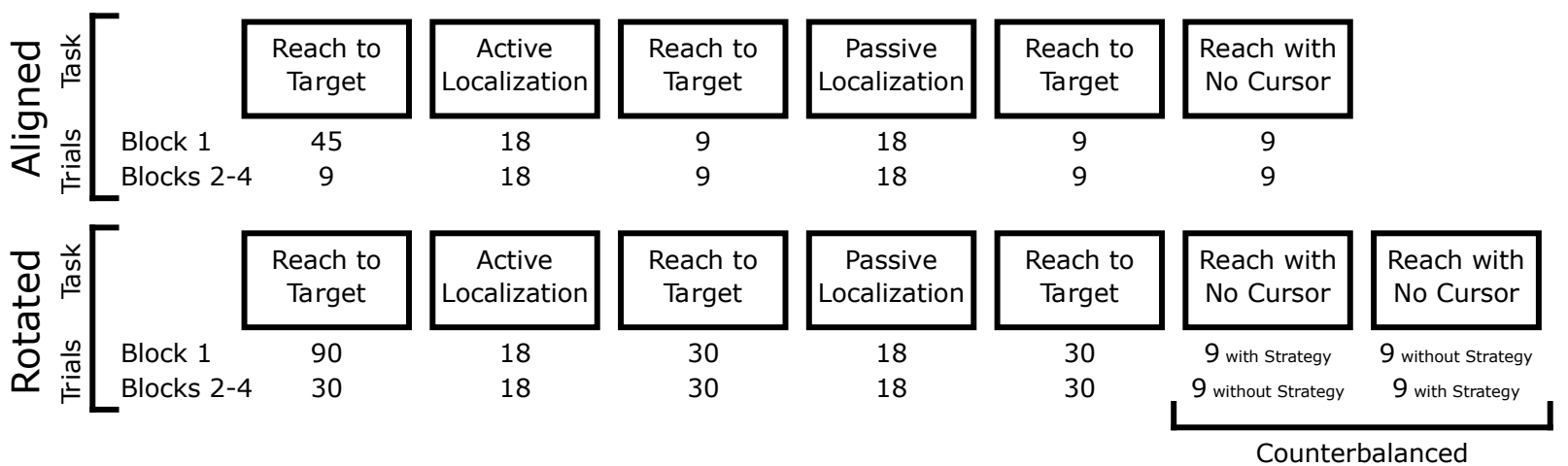

Figure 2. Task order. Top row: First session was the baseline session, where the cursor was aligned with the hand position in reach training tasks. Bottom row: Second session involved training with a rotated cursor. Each session began with reach training and consisted of four tasks (e.g. reach training, active localization, passive localization, and no cursor reaches). Each set of four tasks represents a block, and each block was completed four times, starting with training. This was followed by two hand localization tasks, during which either the participants moved their own unseen hand to the arc (active localization) or the robot guided their hand (passive localization). "Top-up" reach training followed each of these localization tasks. Each block ended with a no-cursor reach task. For the no-cursor reaches following training with a visuomotor rotation in the second session (bottom), participants completed this task twice, once when told to not use their strategy (no-cursor reaches without strategy) and once when told to use it (nocursor reaches with strategy). The order of these two tasks were counterbalanced within and between participants. 
i.e., that they could draw, for at least three times, an arrow on a clock illustrating the direction at which they would reach when using the provided strategy. The noninstructed participants were told that "the reach training tasks will be different as the cursor will not move the same way as it did previously, and they will need to compensate for it by figuring out an appropriate strategy". Both instructed and noninstructed participants were also told they would be called upon to use their strategy during some subsequent reaches and not use the strategy in other reaches (described in detail below).

Localization tasks. The localization tasks assessed the participants' estimated location of their unseen hand following reach training, when they either reached with their hand themselves (active localization) or when the robot displaced their hand (passive localization) to the white arc (Figure 1C; Figure 2). Both active and passive localization allowed the use of afferent information on hand position. Since participants generated a motor command in active localization, they also had efferent information on hand location in this localization task $[18,33,35]$. By measuring both, we can tease apart the contribution of (and changes in) afferent and efferent estimates for hand localization.

In the active localization tasks, participants made a quick and straight hand movement with the robot manipulandum toward a chosen point on a white arc located $12 \mathrm{~cm}$ away from the home position. The arc was $0.5 \mathrm{~cm}$ wide, spanned $60^{\circ}$ and was put in three locations: centred on $50^{\circ}, 90^{\circ}$, or $130^{\circ}$ in polar coordinates (Figure $1 \mathrm{C}$ shows the $50^{\circ}$ position). When the robot handle reached the distance of the arc, a force "cushion" was applied to prevent the participant from moving their hand past the arc, giving them the sensation of hitting a soft wall. After "hitting the wall", participants returned their right hand to the home position (along the same constrained path as in the other tasks), and then used their visible, and untrained left hand to indicate on the touch screen the point where they thought the right hand intersected the arc (Figure 1C). To avoid unwanted contact with the touch screen, participants placed their left hand under their chin between each response. Six hand localization trials were completed at each of the arc locations (for a total of 18 trials) for each of the four repetitions (Figure 2) which sum to a total of 72 trials per session.

In the passive localization tasks, the participants' unseen right hand was pulled by the robot manipulandum to a specific point on the arc on each trial. These points were the endpoints of the reaches generated by the participant in the preceding active localization task. Like in the active localization task, participants returned their hand to the home position along a constrained path and then indicated with their visible left hand, the location of the point on the arc where the right hand had been (Figure 1). This was done for the same number of trials as in the active localization task.

No-cursor reaches. Participants reached to the same three targets as in the training trials (see above) but without visual feedback about the position of their hand (i.e. no cursor). Trials were considered complete when participants had moved away from the home position and indicated that they had acquired the target by holding their hand still for $500 \mathrm{~ms}$. Then, participants moved the robot handle back to the home position, 
through a constrained channel, to begin the next trial. During the first, aligned session, the no-cursor reach tasks involved asking participants to reach to the target nine times each in four tasks (for a total of 36 trials; 12 to each target). During the second, rotated session, these no-cursor trials were split into two sub-tasks (of nine trials each). In one sub-task, participants viewed the words "No-Cursor with Strategy" displayed on the monitor and were required to use the strategy that they also used during their recent training with the blue cursor. In the other subtask, participants saw the words "NoCursor without Strategy" and they were asked to not use a compensatory strategy and reach as they did in the aligned session (as in [20]). The order of these subtasks was counterbalanced between and within each consecutive participant: some started with strategy and some without and this order was inverted in subsequent no-cursor tasks for every participant. Each pair of subtasks was repeated four times (for a total of 72 trials; 24 to each target, 12 of which with and 12 without strategy).

The results of the no-cursor reaches with and without strategy were compared as per a process dissociation procedure (PDP; see [20]), to determine the levels of explicit and implicit learning. The no-cursor reaches without strategy reflect implicit learning (involuntary reach aftereffects) while any additional deviation in the direction of nocursor reaches with strategy reflects explicit leaning. This procedure was used to determine if participants were aware of the nature of the rotation, and could apply the strategy at will, even if they had no visual feedback of their hand. This allowed testing if the instructions lead to explicit learning, and if this was different for the two age groups.

All analyses were done using R 3.4.4 (R Core Team, 2018). All statistical tests used an alpha level of 0.05 , and for ANOVAs where sphericity was violated, the GreenhouseGeisser corrections were used. Partial eta-squares (denoted $\eta^{2}$ ) were used to report effect size for significant effects in ANOVAs. Significant interactions were followed up with a Welch Two Sample t-test (correcting for unequal variances and unequal sample sizes), with eta-squared as effect size (also denoted $\eta^{2}$ ).

\section{Results}

\section{Rotated reach training}

We wanted to test the effect of instruction (instructed or non-instructed) on initial reach adaptation between older and younger adults (Figure 3). To do this we compared the reach deviations during the first three reach training trials, second set of three trials and the final set of 15 trials of the 90 trials in the initial rotated-cursor training task (with the reach deviations from the last 15 trials in the initial aligned training task subtracted). We performed a $2 \times 2 \times 3$ mixed ANOVA with age (younger and older) and instruction (instructed or non-instructed) as between-subject factors and trial set (first, second and final) as a within-subject factor. As shown in Figure 3, all participants' reach deviations, regardless of instruction and age, significantly increased across trial sets (Figure 3B), as required to compensate for the cursor rotation. This was verified by a main effect of trial set, $F(2,150)=94.81, p<0.001, \eta^{2}=0.48$, There was an interaction between trial set and instruction, $F(2,152)=10.45, p<0.001, \eta^{2}=0.082$, showing that instruction affected the time course of adaption. More importantly, there was an interaction 
between age and instruction, $F(1,75)=4.63, p=0.035, \eta^{2}=0.022$, showing that the ability to use instructions or not across trial sets to compensate for the perturbation does depend on whether the participant was older or younger.

As prior research showed that instruction, or explicit strategies, mainly affected adaptation during early training [16,21], we compared age (older and younger) and instruction (instructed and non-instructed) for the initial two sets of trials separately in two follow-up ANOVAs.

For the first trial set, a $2 \times 2$ ANOVA with instruction (instructed and non-instructed) and age (older and younger) on reach deviations. We find a main effect of instruction, $F(1$, $75)=32.32, p<0.001, \eta^{2}=0.301$, as well as an interaction between age and instruction, $F(1,76)=4.72, p=0.033, \eta^{2}=0.059$. In a planned follow-up t-test on reach deviation for the first trial set the older and younger instructed participants showed an effect of age, $t(33.34)=2.71, p=0.011, \eta^{2}=0.16$. That is, we found that reach deviations in instructed older adults (violet line in Figure 3A \& 3B) were one-third smaller than those in instructed younger adults (orange line). A second planned t-test on instructed and non-instructed older adults (Figure $3 \mathrm{~A} \& \mathrm{~B}$, solid blue and dashed purple lines, respectively) shows an effect of instruction within older participants, $t(35.96)=2.06, p=$ $0.047, \eta^{2}=0.106$. In summary, in the first three training trials, reach deviations were larger (greater cursor compensation) for the instructed groups compared to the noninstructed ones, but these were not as large for the instructed older adults compared to the instructed younger adults.

We also tested the age-dependent difference in reach deviations as a function of instruction for the second set of trials using a $2 \times 2$ ANOVA using age (older and

A

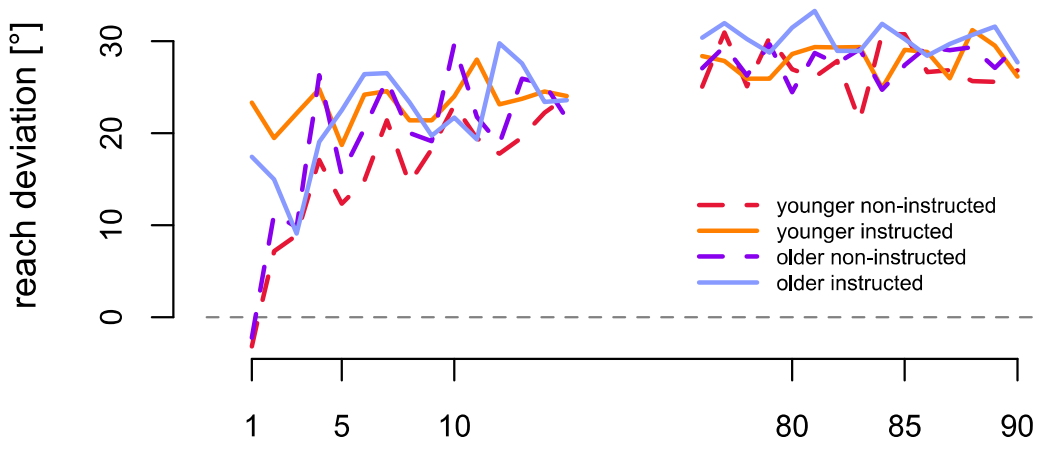

trial
B

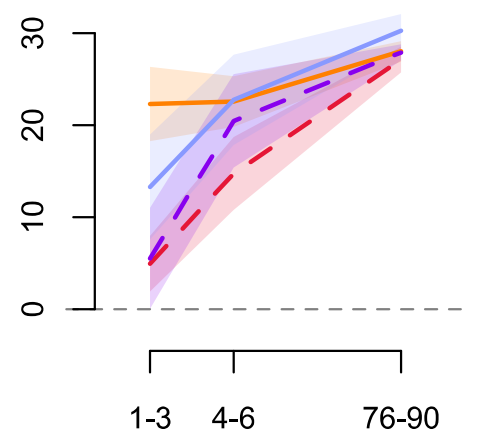

trial set

Figure 3. Learning Curves. Lines represent the mean, baseline-corrected reach deviations for each group with instructed groups as solid lines, and non-instructed groups as dashed lines. Shaded areas represent 95\% confidence intervals. A: Mean reach deviations are shown across the first 90 training trials with rotated cursor. B: The mean across participants and trials within the initial, second and final trial sets. 
younger) and instruction (instructed or non-instructed) as between-subject factors. Instruction still led to overall greater reach deviations, $F(1,75)=6.29, p=0.014, \eta^{2}=$ 0.077 , which suggests instructions still provided a benefit in training trials 4,5 and 6 . However, this benefit of instructions did not vary with age, $F(1,75)=1.89, p=0.17, \eta^{2}$ $=0.024$. And as illustrated in Figure 3 , by the last trial set, all groups attain near perfect compensation (reach deviations of roughly $30^{\circ}$ ).

\section{No-cursor reaches}

No cursor reaches are used to assess cognitive awareness of the cursor rotation developed during training with a rotated cursor both as a function of age, instruction (both factors as above) as well as strategy use (with strategy or without strategy). First, we determined if adapting to a visuomotor rotation lead to changes in no-cursor reach directions, or reach aftereffects, by comparing no-cursor reach directions without strategy from the rotated session with no-cursor reach deviations in the aligned session. As we expected, significant reach aftereffects of approximately $15^{\circ}$ did emerge, $F(1,75)$ $=746.93, \mathrm{p}<0.001, \eta^{2}=0.75$. Given that training with a visuomotor rotation did produce significant changes to no-cursor reaches, i.e. there are reach aftereffects, we continue analyzing no-cursor reach deviations with the aligned no-cursor reach deviations subtracted from both the withstrategy and without-strategy no-cursor reach deviations.

To test our main question about cognitive awareness, we ran a $2 \times 2 \times 2$ ANOVA on reach aftereffects, with age and instruction as between-subjects factors (as used above) and strategy use as within-subjects factor. For those aware of the cursor rotation, the corresponding no-cursor reach deviations when asked to reach with a strategy should be larger than those when asked not to use the strategy. And, for those who are not aware, there should be no difference between these two no-cursor reach tasks. We used this process dissociation procedure (PDP), to determine whether this measure of awareness varied with age. As shown in Figure 4, older adults have, on average, roughly $20 \%$ larger no-cursor reach deviations than younger adults, $F(1,75)=$ 5.66, $p=0.02, \eta^{2}=0.07$. Although everyone had adapted fully during training prior to these no-cursor reaches, i.e. reach

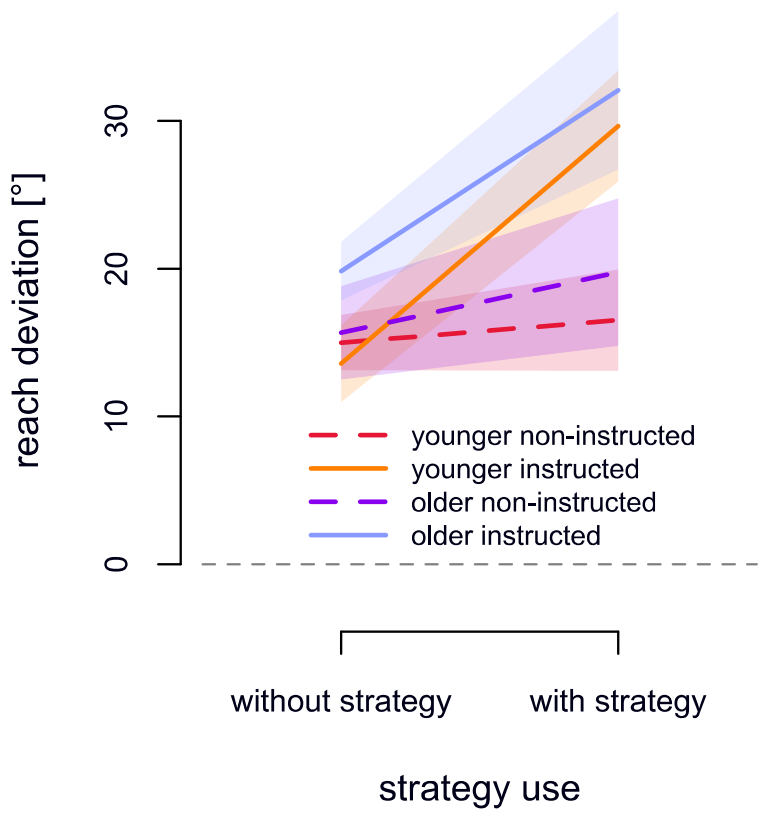

Figure 4. Changes in no-cursor reaches following training. Mean, baseline-corrected reach deviations in no-cursor reaches for each group, i.e, reach aftereffects, while suppressing (left side) or employing (right side) any strategies used during adaptation. Shaded areas and error bars are 95\% confidence intervals. 
deviations of nearly $30^{\circ}$ during training. We also found an interaction between instruction and strategy use, $F(1,75)=35.56, p<0.001, \eta^{2}=0.12$. This suggests that only those given instruction produced larger no-cursor reach deviations when asked to use the strategy (right side of Figure 4) when compared to not using the strategy (left side of Figure 4). More importantly, we found no difference in this pattern between older adults (blue and purple) and younger adults (orange and red), as there was no threeway interaction between age, instruction and strategy use on no-cursor reach deviations, $F(1,75)=2.86, p=0.09, \eta^{2}=0.012$. This suggests that the effect of instruction on awareness was equal for both age groups.

In other words, despite smaller reach deviations for instructed older adults during the first trial set in rotated training, instructed older adults did evoke the strategy during the no-cursor reaches when asked. Crucially, it seems that older instructed adults could evoke their explicit strategy to the same extent as younger instructed adults. This means that instructions evoked explicit learning and they did so equally for younger and older adults.

\section{Hand localization}

Now we can test if afferent and efferent estimates of hand location vary as a function of explicit learning and if this is different for older and younger participants (Figure 5). First, we have to confirm that training with a rotated cursor induces shifts in hand localization using a four-way $2 \times 2 \times 2 \times 2$ mixed ANOVA that includes training session (aligned and rotated session) and movement type (active and passive localization) as within-subject factors, and age (older and younger) and instruction (instructed and non-instructed) as between-subjects factors. There is a main effect of training, $F(1,75)=231.34, p<.001$, $\eta^{2}=0.28$. This means that the estimated location of the unseen hand in the second session is significantly shifted relative to the first, and in the direction expected after training with a CCW rotated cursor. Moreover, training session significantly interacts with movement type, $F(1,75)=10.01, p=.002, \eta^{2}=0.002$. Thus, the results replicate prior findings [33] of a significant effect of movement type; that is, the training-induced shifts in hand localization are larger for active localization than for passive localization, confirming an additional contribution to state estimates of hand location from updated efferent-based predictions of sensory consequences in active localization.

Given that the rotated cursor training leads to a significant change in hand position estimates, which in turn varies with movement type, the next step was to test whether age and instruction affect this pattern. First, like for the reach aftereffects, we took the difference in localization between the rotated session and the aligned session, to calculate hand localization shifts for both the active and passive localization tasks (as plotted in Figure 5A and 5B). We test whether these shifts in hand-localization vary as a function of age and instruction, using a $2 \times 2$ ANOVA for passive localization, where only afferent (proprioceptive) information was available (Figure 5B). Older adults (purple lines) produce significantly larger shifts in the afferent-based estimates of hand position, almost double that of younger adults (orange lines), $F(1,75)=10.01, p=0.002, \eta^{2}=$ 0.118 , but there is no main effect of instruction, $F(1,75)=0.02, p=0.87, \eta^{2}<0.001$, 

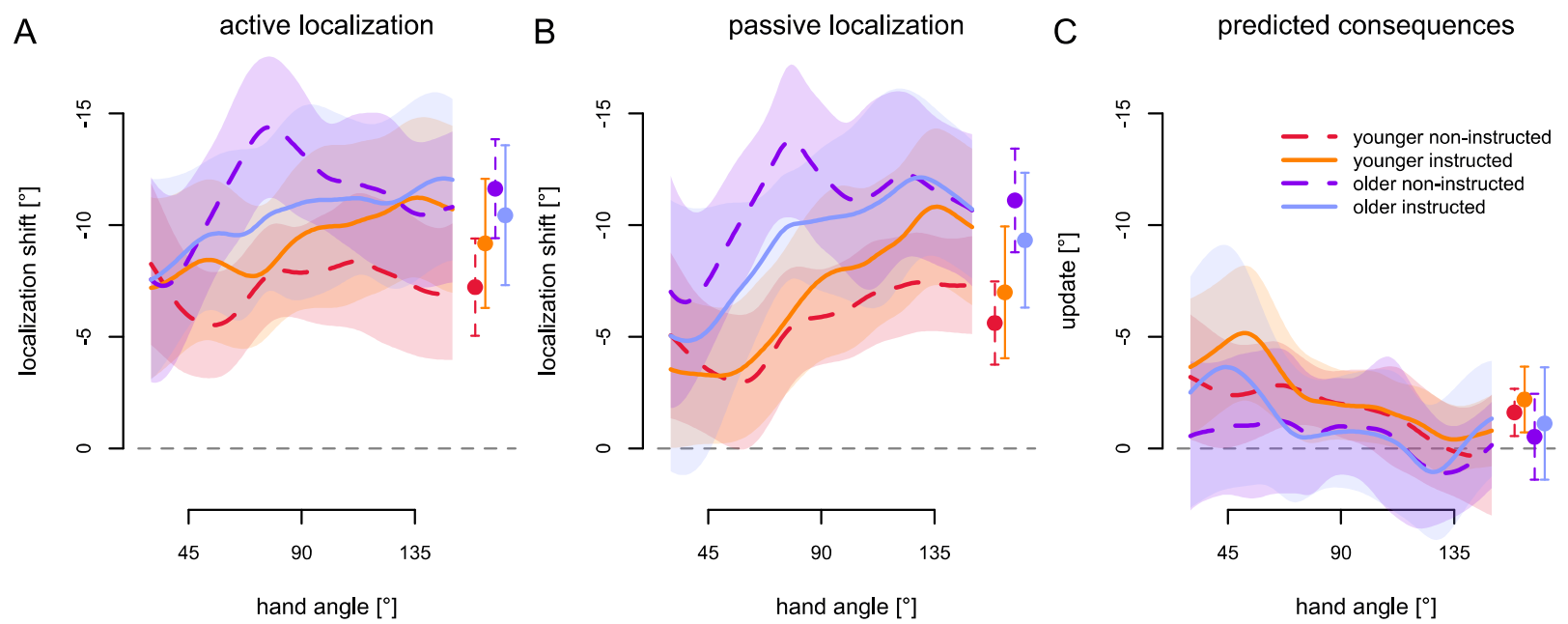

Figure 5. Training-induced shifts in hand localization. A: Active localization: mean shift in hand localization estimates after training where participants actively moved their unseen right hand to a location of their choosing on the white arc (efferent and afferent). $B$ : Passive Localization: Mean shift in hand localization estimates after training where the unseen right hand is passively moved to the exact location as the active localization task (only afferent). C: Predicted sensory consequences: Differences in estimates between active hand localization (efferent and afferent) and passive hand localization (afferent only) are interpreted as evidence there are changes in efferent-based estimates of hand location. Shaded areas and bars are $95 \%$ confidence intervals.

suggesting that explicit learning does not affect afferent hand localization signals. Lastly, there is no interaction between instruction and age, $F(1,75)=1.63, p=0.21, \eta^{2}$ $=0.021$. Thus, in summary, while older adults show larger shifts in afferent-only hand localization, or proprioceptive recalibration, there is no effect of instruction on proprioceptive recalibration, confirming this is a wholly implicit process [18].

As indicated earlier, the shifts in hand localization are larger in active localization (Figure 5A) than in passive localization (Figure 5B), likely because this task also includes changes due to updated efferent-based or predicted estimates of hand position. However, when we try to isolate the updated predicted or efferent-based estimates by subtracting shifts in hand localization responses in the passive condition from those in the active condition (Figure $5 \mathrm{C}$ ), the difference and the effect size is small overall. This suggests that the training-induced changes in hand estimates in the active localization task (Figure 5A) might be primarily due to proprioceptive recalibration (Figure 5B).

Further, as in the original $2 \times 2 \times 2 \times 2$ ANOVA a $2 \times 2$ ANOVA on the difference between active and passive localization shifts (as in Figure $5 \mathrm{C}$ ) using age and instruction as between-subject factors, we find no effect of age, $\mathrm{F}(1,75)=1.59, p=0.19, \eta^{2}=0.02$ (nor any other effect or interaction). However, when we compare the magnitude of these updated predicted shifts in hand position for each age group to zero, older adults show only $0.84^{\circ}$ shift, which did not significantly differ from $0, t(35)=1.07, p=0.15, \eta^{2}=$ 
0.032 . However, the shifts for younger adults, $1.97^{\circ}$, are significantly different from 0 , $t(41)=4.62, p<.001, \eta^{2}=0.481$, as found by 't Hart \& Henriques [33]. These results are consistent with the confidence intervals shown in Figure 5C. In summary, while older adults show much greater shifts in afferent-based hand localization, or proprioceptive recalibration, than younger adults, they do not show evidence of a contribution from updates to efferent-based predictions in hand localization.

\section{Discussion}

In this study, we assess the effects of age-related changes in explicit and implicit processes on motor learning to determine whether age-related deficits in visuomotor learning are explained by these processes. Indeed, we find clear evidence for agerelated deficits in "explicit" aspects of adaptation but not for implicit aspects. Specifically, we find that older adults benefit less from explicit instruction during initial adaptation despite including their strategy to the same extent as younger adults when reaching without a cursor. In contrast, we find no impairments in implicit changes for older compared to younger adults that would likely contribute to age-related declines in motor learning. In fact, older adults show marginally larger reach aftereffects and clearly larger proprioceptive recalibration compared to younger adults suggesting implicit processes are maintained or even enhanced with age. We find no age-related differences for learning-induced changes in efferent-based estimates of the hand. However, the added effect of the presence of efferent-based signals on hand localization shifts are so small as to be non-detectable in older adults, but not younger adults. In summary, while we find some suggestion of age-related deficits in the use of an explicit strategy, we find none related to implicit changes, and in fact, find that implicit motor and proprioceptive changes were larger in older adults.

\section{Explicit component of adaptation in aging}

Our findings replicate prior research that demonstrates that instruction on how to counteract a perturbation can benefit initial adaptation [16,17,19-21]. Using the same instructions as in the current study on only younger adults, Werner et al., [20], Benson et al., [21], Neville et al, [19] and Modchalingam et al. [18] we find that instructed participants compensate more for the cursor-rotation than non-instructed younger adults at the start of training. In the current study, we find that the extent of this benefit varies as a function of age. That is, while instructed older adults show significantly more compensation during initial learning compared to non-instructed older adults, this benefit is $1 / 3$ smaller than for instructed younger adults (Figure 3 ). Other studies also find that older adults benefit less from instruction. For example, Heuer \& Hegele $[4,17]$ demonstrate that, when both given instruction and corrective feedback, older adults are less able to acquire or apply a cognitive strategy when training to rotate an arrow toward the direction they would have to move, to compensate for a $75^{\circ}$ visuomotor rotation. However, it is unclear at what point in time differences in the acquisition and application of an adaptive strategy emerge during learning, as they average out performance across trials. Here, the age-related differences in applying a cognitive strategy for a smaller rotation of $30^{\circ}$ are limited to only the initial stages of learning (specifically the 
first set of 3 trials). This suggests that the ability or willingness to adopt a novel explicit strategy decreases with age. This finding supports the hypothesis that age-related cognitive or explicit changes can lead to a deficit in initial motor learning under certain perturbations.

Other age-related deficits can emerge even in the absence of instruction. However, the present study shows no differences in initial learning between non-instructed older and younger adults. The difference between the current results and those showing an agerelated deficit in visuomotor adaptation could be attributed to the size of the rotation and its implication on cognitive awareness of the visual perturbation $[19,20]$. Studies that use larger perturbations (greater than $45^{\circ}$ ) are more likely to find age-related differences for learning rate or learning extent $[2-7,10,51]$. For smaller rotations (e.g. $30^{\circ}$ rotation) the findings are less consistent: some studies find older adults adapt less than younger adults $[12,51]$ and others, like the current study, find no such age-related differences $[4,7]$ in the absence of instruction. Thus, it may be that with larger perturbations, cognition or explicit processes are more likely to be engaged and play a larger role [1820], and thus, any deficits in using a strategy are more likely to emerge. Alternatively, smaller perturbations may rely on more implicit processes that appear to be intact in older adults. Thus, these conflicting findings regarding age-related differences in motor learning can be mainly attributed to perturbations or tasks that are more likely to solicit explicit strategies.

Given the small perturbation used in the current study, it is not surprising that older adults did not show any differences in the level of awareness or the ability to produce a strategy post-training when compared to younger adults. This is objectively assessed using the process dissociation procedure (PDP) also used by $([19,20])$ and indirectly by $[4,17]$. In their studies and ours, those who are told how to counter the cursor-rotation or who became aware of the nature of the perturbation in another way are able to evoke the strategy, that is produce larger reach deviations, when asked. In the present study, while both non-instructed and instructed groups compensate fully for the relatively small cursor rotation, only those who received instructions produce this PDP disassociation pattern indicative of strategy use. This is true for both older and younger adults, despite the initial adaptation differences between instructed older and younger adults. However, this absence of a difference in PDP-measured awareness may again be related to the size of the perturbation. Others have demonstrated that larger rotations, such as a $60^{\circ}$ rotation [18-20] or a $75^{\circ}$ rotation $[4,17]$, even in the absence of instruction, can lead to PDP awareness in younger adults after training. Moreover, Heuer and Hegele $[4,17,27]$ find that fewer older adults show this disassociation, and when they do it is smaller and generalizes less, suggesting that older adults are less able to develop and use the strategy for this larger rotation. These findings suggest that while the development and use of a cognitive strategy during adaptation may be impaired in older adults [2], this impairment scales with cognitive demand as in when the size of the perturbation is greater. Whereas for less difficult, e.g. smaller, perturbations as in the current study, older adults can and do understand the instruction and apply them later in training, but only partially early on in training. 


\section{Cognitive ability and motor learning}

Consistent with the idea that age-related differences in adaptation may be due to deficits in cognitive or explicit processes, several studies show a relationship between age-related motor learning decline and cognitive tasks that measure spatial working memory [1,52], divergent/logic thinking [53], and inhibition [54]. Using a force-field adaptation paradigm, Trewartha et al [9] find no age-related differences in performance during training but suggest that age-related cognitive changes in declarative (spatial) memory do contribute to small age-related differences in the components of motor learning. Simon \& Bock [54] find that adapting to a $60^{\circ}$ visuomotor rotation is correlated with inhibition, as measured by Stroop scores, throughout the motor learning task in older adults; in contrast, for younger adults, the correlation with inhibition decreases over time. These authors $[2,53,54]$ suggest that cognitive contribution or strategical control in adaptation are more impaired for older adults, which can explain differences in motor learning with age. Exploring the relationship between cognition and motor learning, Christou et al., [29] show that a small portion of younger participants with poor spatial working memory (SWM) also show a smaller explicit contribution in their learning. Likewise, Anguera et al., [1] find that some SWM tasks do correlate with initial adaptation rate to a $30^{\circ}$ visuomotor rotation in younger adults, but not for older adults even though this older group adapts more slowly. Moreover, they find that while younger adults show overlapping neural activities in the dorsal lateral frontal cortex during the early adaptation period and SWM performance, older adults do not show a similar significant neural overlap. Given the possible importance of SWM in initial visuomotor learning, Anguera et al., [1] suggest that the lack of activation of SWMrelated brain areas in older adults during visuomotor adaptation, despite normal brain activity in other tasks, may be related to their failure to effectively engage SWM processes during learning. This in turn could explain age-related deficits in initial visuomotor adaptation because they might be less able to mentally rotate the actual hand position with the rotated visual hand position while reaching, which could be important for explicit strategies. Another group of researchers, Wong et al., [52] encourage the development of a cognitive strategy to compensate for a $45^{\circ}$ visuomotor rotation by showing the actual hand and a landmark target as well as the reaching target and rotated cursor during adaptation in cerebellar patients, older controls, and younger adults. They find that older adults and patients adapt slower, and to a lesser extent, regardless of whether the actual hand is visible or not. The effect of having the hand visible on learning is slightly correlated with Digit Span (working) memory, but not with the visuospatial task they use, for the two older groups. Overall, these studies suggest that some cognitive tasks, which may not necessarily involve only spatial components, are correlated with poorer performance in both older and younger adults. In their review of this literature, Buszard \& Masters, [55] suggest that indeed the evidence relating cognitive performance and adaptation performance is weak (although stronger for sequence learning). Nonetheless a possible association between cognitive and adaptation performance is consistent with our small age-related effect of instruction on early adaptation. And yet while not clearly demonstrated, it cannot be ruled out that age-related changes in cognition $[30,31,56]$ which also includes working memory, may lead older participants to benefit less from instructions when initially adapting to the 
visuomotor rotation.

\section{Proprioceptive recalibration and aging}

As demonstrated in this study and others, there is some suggestion that explicit processes decline with age, but it is not clear whether implicit processes are similarly affected. In this study, we attempt to assess implicit processes by measuring implicit reach aftereffects (no-cursor reaches without strategy) as well as the change in hand position estimates. Both of which have been shown to be unaffected by instruction or cognitive strategy $[16,18]$ and are similar in magnitude whether the distortion is introduced gradually or abruptly (as demonstrated across different studies using the same setup; e.g., [46,57]). We find that reach aftereffects are not lower and may even be slightly larger in older adults than younger adults, suggesting that implicit processes are intact. Further, we replicate that reach training with altered visual feedback of the hand leads to shifts in the perceived hand position towards the prior visual experience $[15,34-37,41,43,44]$. Unsurprisingly, we partially replicate the finding that proprioceptive recalibration occurs independent of age [15]. This prior study from our lab demonstrates that both older and younger adults shift the position of their felt hand to coincide with a reference marker, after training with a cursor gradually rotated to $30^{\circ}$, using a twoalternative forced choice method. In this earlier study, both age groups recalibrate their proprioception roughly $6^{\circ}$, i.e., approximately $20 \%$ of the visuomotor distortion. However, our findings show a moderately larger recalibration with age $\left(6.3^{\circ}\right.$ for younger, and $10.2^{\circ}$ for older adults; see Figure 5B). Using our passive localization task, we find that older adults produce larger shifts in hand estimates compared to younger adults. Likewise, we also find slightly larger reach aftereffects in this older group. The passive localization task in our study and the perceptual measure of Cressman et al., [15] produces similarly sized changes in proprioception in younger adults [34]; however, it is possible that it does not for older adults. Plus, it appears the faster method of measuring proprioceptive change we use here is less vulnerable to decay (compare $[37,58]$ ). Thus, it may be that age-related differences are more detectable in the passive localization task than in Cressman et al., [15]. Apart from the methods, Cressman et al., [15] also have fewer older participants (9 vs. our 38) that are slightly younger (average 66 years vs. 70 years). Consequently, increasing the sample size by roughly four times, combined with using a slightly older population in this study perhaps provides the necessary power to detect a difference between age groups. Nonetheless, we find that the implicit change in proprioceptive hand estimates following learning is at least not smaller than in younger adults, and therefore cannot explain possible age-related decline in adaptation.

While our results suggest that these implicit sensory changes cannot explain agerelated differences in adaptation, it is not clear why older adults are showing larger proprioceptive recalibration. One explanation could be that the larger proprioceptive hand shifts or recalibration in older adults are due to a greater reliance on visual over proprioceptive feedback. However, this does not seem to be a good explanation for our results. Findings from Block \& Bastian, [59,60] show no relationship between sensory weighing and sensory realignment following training with visual-proprioceptive 
mismatches in healthy, younger adults [60] and in cerebellar patients as well as their age-matched (i.e. older) controls [59]. In addition, other studies measuring estimates of final hand positions also fail to find a correlation between hand estimates using multiple modalities and the precision or relative reliability of either visual or proprioceptive-only estimates of location [61-63]. While there are studies that suggest that proprioceptive acuity is poorer in older adults than younger adults (see [64] for a review), including one that tests felt hand position in our own lab [15], the current study, with our much larger sample of participants and larger number of proprioceptive trials, does not find that older adults are less precise in their estimates of hand position prior to training with a rotated cursor [65]. Moreover, when compared across all these older and younger adults, the size of proprioceptive recalibration does not correlate at all with the amount of variance in hand-estimates [65]. Together, these studies demonstrate that a possibly greater reliance on vision resulting in greater proprioceptive recalibration in older adults cannot simply be explained by poorer proprioceptive sensitivity. Further studies are required to understand the factors that influence the amount of proprioceptive recalibration.

\section{Updated prediction with age}

Motor learning does not only lead to changes in implicit processes such as proprioceptive estimates of hand position, but also to updates in predicted sensory consequences - a required change to produce implicit adaptation. 't Hart and Henriques [33] found that proprioception accounted for at least half of learning-induced changes in hand localization (as originally found by $[48,49]$ using only an active localization task), compared to prediction. This partitioning out of predictive and proprioceptive based change is consistent with the results of the cerebellar patients in the studies by Izawa et al., [49] and Synofzik et al., [48]. Both studies find that the patients show a significant change in active hand localization following adaptation, but that the change in hand estimates is smaller than in healthy controls. Both studies interpret their result to support the idea that cerebellum is critical for updating the predicted consequences of movements during learning. This leaves open the possibility that the remaining shift in the cerebellar patients may be due to proprioceptive recalibration, which our lab has shown to also occur with cerebellar patients [44]. In the current study, the relative proportion of predicted and perceived changes in hand localization was even more skewed than those of 't Hart and Henriques [33] (despite more targets and trials), with younger adults showing a change in hand localization: $80 \%$ of which was due to a change in proprioception and $20 \%$ a change in prediction. The change in predictive estimates of hand position is further reduced in older adults, such that the $\sim 1^{\circ}$ change is not statistically detectable (although their proprioceptive change is $50 \%$ larger than that of younger adults). This difference is not significant relative to younger adults since the changes for younger adults are also quite small; approximately $20 \%$ of the changes reflecting only a 2 to $3^{\circ}$ shift (Figure 5). It may be that the proprioceptive recalibration of larger magnitudes in older adults may act as a ceiling effect or generally mask any further change due to prediction. Nonetheless, given that there is no age-related effect on these updated predicted estimates - which are implicit by nature and not affected by instruction (as shown in [18]) - updates in predicted estimates likely do not explain possible age-related effects on motor adaptation. 


\section{Conclusion}

This study demonstrates that age-related decline leads to a reduced benefit of instruction (smaller reach deviations) in early reach adaptation. This suggests that cognitive changes with age can contribute somewhat to the decreased acquisition and application of a strategy to initially adapt. However, the overall extent of adaptation is not affected in older adults, in that non-instructed older and younger adults adapt at similar rates. We also show implicit changes such as shifts in hand estimates (proprioceptive recalibration) and reach aftereffects in older adults are greater than in younger adults after training with a visuomotor rotation, independent of their awareness of this rotation. This greater proprioceptive recalibration with older age may be due to greater reliance on vision over proprioception; perhaps due to decline in proprioceptive acuity. However, we find that aging does not affect the updating of predicted sensory consequences after training with altered visual feedback. Altogether, these findings suggest that the explicit contribution to motor learning decreases with age, whereas the implicit processes appear to remain intact. This study shows that aging may lead to subtle, yet important changes, in sensorimotor learning and multisensory integration.

\section{Acknowledgements}

This work was supported by a Canadian Network for Research and Innovation in Machining Technology NSERC Operating grant (DYPH) and the German Research Foundation (DFG) under grant no. HA 6861/2-1 (BMtH). The funders had no role in study design, data collection and analysis, decision to publish, or preparation of the manuscript.

\section{References}

1. Anguera JA, Reuter-Lorenz PA, Willingham DT, Seidler RD. Failure to Engage Spatial Working Memory Contributes to Age-related Declines in Visuomotor Learning. J Cogn Neurosci. 2011;23: 11-25. doi:10.1162/jocn.2010.21451

2. Bock $\mathrm{O}$, Girgenrath $\mathrm{M}$. Relationship between sensorimotor adaptation and cognitive functions in younger and older subjects. Exp Brain Res. 2006;169: 400-406. doi:10.1007/s00221-005-0153-4

3. Buch, E. R.; Young, S.; Contreras-Vidal JL. Visuomotor Adaptation in Normal Aging. Learn Mem. 2003;10: 55-63. doi:10.1101//m.50303

4. Heuer H, Hegele M. Adaptation to Visuomotor Rotations in Younger and Older Adults. Psychol Aging. 2008;23: 190-202. doi:10.1037/0882-7974.23.1.190

5. Heuer H, Hegele M, Sülzenbrück S. Implicit and explicit adjustments to extrinsic visuo-motor transformations and their age-related changes. Hum Mov Sci. Elsevier B.V.; 2011;30: 916-930. doi:10.1016/j.humov.2010.07.004

6. King BR, Fogel SM, Albouy G, Doyon J. Neural correlates of the age-related changes in motor sequence learning and motor adaptation in older adults. Front Hum Neurosci. Frontiers Media SA; 2013;7: 142. doi:10.3389/fnhum.2013.00142 
7. Seidler RD. Differential effects of age on sequence learning and sensorimotor adaptation. Brain Res Bull. 2006;70: 337-346. doi:10.1016/j.brainresbull.2006.06.008

8. Huang HJ, Ahmed AA. Older adults learn less, but still reduce metabolic cost, during motor adaptation. J Neurophysiol. 2014;111: 135-144. doi:10.1152/jn.00401.2013

9. Trewartha KM, Garcia A, Wolpert DM, Flanagan JR. Fast But Fleeting: Adaptive Motor Learning Processes Associated with Aging and Cognitive Decline. J Neurosci. 2014;34: 13411-13421. doi:10.1523/JNEUROSCI.1489-14.2014

10. Fernández-Ruiz J, Hall C, Vergara P, Díaz R. Prism adaptation in normal aging: Slower adaptation rate and larger aftereffect. Cogn Brain Res. 2000;9: 223-226. doi:10.1016/S0926-6410(99)000579

11. Roller CA, Cohen HS, Kimball KT, Bloomberg JJ. Effects of normal aging on visuo-motor plasticity. Neurobiol Aging. 2002;23: 117-123. doi:10.1016/S0197-4580(01)00264-0

12. Lei $\mathrm{Y}$, Wang J. The effect of proprioceptive acuity variability on motor adaptation in older adults. Exp Brain Res. Springer Berlin Heidelberg; 2017;0: 1-10. doi:10.1007/s00221-017-5150-x

13. Cressman EK, Henriques DYP. Reach adaptation and proprioceptive recalibration following exposure to misaligned sensory input. J Neurophysiol. 2010;103: 1888-1895. doi:10.1152/jn.01002.2009

14. Hegele $M$, Heuer $\mathrm{H}$. Adaptation to a direction-dependent visuomotor gain in the young and elderly. Psychol Res Psychol Forsch. Springer-Verlag; 2010;74: 21-34. doi:10.1007/s00426-008-0221-z

15. Cressman EK, Salomonczyk D, Henriques DYP. Visuomotor adaptation and proprioceptive recalibration in older adults. Exp Brain Res. 2010;205: 533-44. doi:10.1007/s00221-010-2392-2

16. Taylor JA, Krakauer JW, Ivry RB. Explicit and Implicit Contributions to Learning in a Sensorimotor Adaptation Task. J Neurosci. 2014;34: 3023-3032. doi:10.1523/JNEUROSCI.3619-13.2014

17. Hegele M, Heuer H. Age-Related variations of visuomotor adaptation result from both the acquisition and the application of explicit knowledge. Psychol Aging. 2013;28: 333-339. doi:10.1037/a0031914

18. Modchalingam S, Vachon C, 't Hart BM, Henriques D. The effects of awareness of the perturbation during motor adaptation on hand localization. PsyArXiv; 2018; doi:10.31234/OSF.IO/ Y53C2

19. Neville KM, Cressman EK. The influence of awareness on explicit and implicit contributions to visuomotor adaptation over time. Exp Brain Res. Springer Berlin Heidelberg; 2018;236: 20472059. doi:10.1007/s00221-018-5282-7

20. Werner S, Van Aken BC, Hulst T, Frens MA, Van Der Geest JN, Strüder HK, et al. Awareness of sensorimotor adaptation to visual rotations of different size. PLoS One. 2015;10: 1-18. doi:10.1371/journal.pone.0123321

21. Benson BL, Anguera JA, Seidler RD. A spatial explicit strategy reduces error but interferes with sensorimotor adaptation. J Neurophysiol. American Physiological Society Bethesda, MD; 2011;105: 2843-2851. doi:10.1152/jn.00002.2011

22. Keisler A, Shadmehr R. A shared resource between declarative memory and motor memory. J Neurosci. 2010;30: 14817-23. doi:10.1523/JNEUROSCI.4160-10.2010 
23. Taylor JA, Thoroughman KA. Motor Adaptation Scaled by the Difficulty of a Secondary Cognitive Task. Robertson E, editor. PLoS One. Public Library of Science; 2008;3: e2485. doi:10.1371/journal.pone.0002485

24. Taylor JA, Thoroughman KA. Divided Attention Impairs Human Motor Adaptation But Not Feedback Control. J Neurophysiol. American Physiological Society; 2007;98: 317-326. doi:10.1152/jn.01070.2006

25. Bedard P, Song J-H. Attention modulates generalization of visuomotor adaptation. J Vis. 2013;13: 12. doi: $10.1167 / 13.12 .12$

26. Im HY, Bédard $\mathrm{P}$, Song J-H. Encoding attentional states during visuomotor adaptation. J Vis. Association for Research in Vision and Ophthalmology; 2015;15: 20. doi:10.1167/15.8.20

27. Heuer $\mathrm{H}$, Hegele M. Generalization of implicit and explicit adjustments to visuomotor rotations across the workspace in younger and older adults. J Neurophysiol. 2011;106: 2078-2085. doi:10.1152/jn.00043.2011

28. Heuer $\mathrm{H}$, Hegele M. Adaptation to direction-dependent visuo-motor rotations and its decay in younger and older adults. Acta Psychol (Amst). 2008;127: 369-381. doi:10.1016/j.actpsy.2007.07.006

29. Christou AI, Miall RC, McNab F, Galea JM. Individual differences in explicit and implicit visuomotor learning and working memory capacity. Sci Rep. Nature Publishing Group; 2016;6: 1-13. doi:10.1038/srep36633

30. Salthouse TA. Shared and unique influences on age-related cognitive change. Neuropsychology. NIH Public Access; 2017;31: 11-19. doi:10.1037/neu0000330

31. Salthouse TA. The processing-speed theory of adult age differences in cognition. Psychol Rev. 1996;103: 403-428. doi:10.1037/0033-295X.103.3.403

32. Barkley V, Salomonczyk D, Cressman EK, Henriques DYP. Reach adaptation and proprioceptive recalibration following terminal visual feedback of the hand. Front Hum Neurosci. 2014;8: 705. doi:10.3389/fnhum.2014.00705

33. 't Hart BM, Henriques DYP. Separating predicted and perceived sensory consequences of motor learning. PLoS One. 2016;11: e0163556. doi:10.1371/journal.pone.0163556

34. Clayton HA, Cressman EK, Henriques DYP. The effect of visuomotor adaptation on proprioceptive localization: The contributions of perceptual and motor changes. Exp Brain Res. 2014;232: 20732086. doi:10.1007/s00221-014-3896-y

35. Cameron BD, Franks IM, Inglis JT, Chua R. The adaptability of self-action perception and movement control when the limb is passively versus actively moved. Conscious Cogn. Academic Press; 2012;21: 4-17. doi:10.1016/J.CONCOG.2010.11.006

36. Cressman EK, Henriques DYP. Sensory recalibration of hand position following visuomotor adaptation. J Neurophysiol. 2009;102: 3505-3518. doi:10.1152/jn.00514.2009

37. Ruttle JE, Cressman EK, Marius 't Hart B, Henriques DYP. Time Course of Reach Adaptation and Proprioceptive Recalibration during Visuomotor Learning. PLoS One. 2016; doi:10.1371/journal.pone.0163695

38. Mostafa AAAA, Kamran-Disfani R, Bahari-Kashani G, Cressman EKEK, Henriques DYPDYP, Kamran-disfani R, et al. Generalization of reach adaptation and proprioceptive recalibration at 
different distances in the workspace. Exp Brain Res. 2014;233: 817-827. doi:10.1007/s00221014-4157-9

39. Ostry DJ, Darainy M, Mattar AAG, Wong J, Gribble PL. Somatosensory Plasticity and Motor Learning. J Neurosci. 2010;30: 5384-5393. doi:10.1523/JNEUROSCI.4571-09.2010

40. Ostry DJ, Gribble PL. Sensory Plasticity in Human Motor Learning. Trends Neurosci. Elsevier Ltd; 2016;39: 114-123. doi:10.1016/j.tins.2015.12.006

41. Mostafa AA, Kamran-disfani R, Bahari-kashani G, Cressman EK, Henriques DYP. Generalization of reach adaptation and proprioceptive recalibration at different distances in the workspace. Exp Brain Res. 2014;233: 817-827. doi:10.1007/s00221-014-4157-9

42. Salomonczyk D, Cressman EK, Henriques DYP. The role of the cross-sensory error signal in visuomotor adaptation. Exp Brain Res. 2013;228: 313-325. doi:10.1007/s00221-013-3564-7

43. Ruttle JE, 't Hart BM, Henriques DYP. The fast contribution of visual-proprioceptive discrepancy to reach aftereffects and proprioceptive recalibration. PLoS One. Public Library of Science; 2018;13: e0200621. doi:10.1371/JOURNAL.PONE.0200621

44. Henriques DYP, Filippopulos F, Straube A, Eggert T. The cerebellum is not necessary for visually driven recalibration of hand proprioception. Neuropsychologia. 2014;64: 195-204.

doi:10.1016/j.neuropsychologia.2014.09.029

45. Mostafa AA, 't Hart B, Henriques DY. Motor Learning Without Moving: Proprioceptive and Predictive Hand Localization After Passive Visuoproprioceptive Discrepancy Training. bioRxiv. 2018;

46. Salomonczyk D, Henriques DYP, Cressman EK. Proprioceptive recalibration in the right and left hands following abrupt visuomotor adaptation. Exp Brain Res. 2012;217: 187-196. doi:10.1007/s00221-011-2985-4

47. Haith AM, Krakauer JW. Theoretical models of motor control and motor learning. Routledge Handb Mot Control Mot Learn London Routledge. 2013; 7-28. Available: http://blam-lab.org/wpcontent/uploads/2013/01/HaithKrakauer_TheoreticalModels-1.pdf

48. Synofzik M, Lindner A, Thier P. The Cerebellum Updates Predictions about the Visual Consequences of One's Behavior. Curr Biol. 2008;18: 814-818. doi:10.1016/j.cub.2008.04.071

49. Izawa J, Criscimagna-hemminger SE, Shadmehr R. Cerebellar Contributions to Reach Adaptation and Learning Sensory Consequences of Action. J Neurosci. 2012;32: 4230-4239. doi:10.1523/JNEUROSCI.6353-11.2012

50. Mostafa AA, Salomonczyk D, Cressman EK, Henriques DYP. Intermanual transfer and proprioceptive recalibration following training with translated visual feedback of the hand. Exp Brain Res. 2014;232. doi:10.1007/s00221-014-3833-0

51. Anguera JA, Reuter-Lorenz PA, Willingham DT, Seidler RD. Contributions of Spatial Working Memory to Visuomotor Learning. J Cogn Neurosci. 2010;22: 1917-1930. doi:10.1162/jocn.2009.21351

52. Wong AL, Marvel CL, Taylor JA, Krakauer JW. Can patients with cerebellar disease switch learning mechanisms to reduce their adaptation deficits? Brain. 2019;142: 662-673. doi:10.1093/brain/awy334

53. Simon A, Bock O. Influence of divergent and convergent thinking on visuomotor adaptation in 
young and older adults. Hum Mov Sci. Elsevier B.V.; 2016;46: 23-29.

doi:10.1016/j.humov.2015.11.020

54. Simon A, Bock O. The role of cognition for different stages of visuomotor adaptation in younger and older adults. Hum Mov Sci. Elsevier B.V.; 2017;52: 215-222.

doi:10.1016/j.humov.2017.02.011

55. Buszard T, Masters RSW. Adapting, correcting and sequencing movements: does workingmemory capacity play a role? Int Rev Sport Exerc Psychol. Routledge; 2018;11: 258-278. doi:10.1080/1750984X.2017.1323940

56. Grady CL, Springer M V., Hongwanishkul D, Mclntosh AR, Winocur G. Age-related Changes in Brain Activity across the Adult Lifespan. J Cogn Neurosci. 2006;18: 227-241. doi:10.1162/jocn.2006.18.2.227

57. Salomonczyk D, Cressman EK, Henriques DYP. Proprioceptive recalibration following prolonged training and increasing distortions in visuomotor adaptation. Neuropsychologia. 2011;49: 30533062. doi:10.1016/j.neuropsychologia.2011.07.006

58. Zbib B, Henriques DYP, Cressman EK. Proprioceptive recalibration arises slowly compared to reach adaptation. Exp Brain Res. 2016;234: 2201-13. doi:10.1007/s00221-016-4624-6

59. Block HJ, Bastian AJ. Cerebellar involvement in motor but not sensory adaptation. Neuropsychologia. Elsevier; 2012;50: 1766-1775. doi:10.1016/j.neuropsychologia.2012.03.034

60. Block HJ, Bastian AJ. Sensory weighting and realignment: independent compensatory processes. J Neurophysiol. American Physiological Society; 2011;106: 59-70. doi:10.1152/jn.00641.2010

61. Mikula L, Gaveau V, Pisella L, Khan AZ, Blohm G. Learned rather than online relative weighting of visual-proprioceptive sensory cues. J Neurophysiol. 2018;119: 1981-1992.

doi:10.1152/jn.00338.2017

62. Jones SAH, Fiehler K, Henriques DYP. A task-dependent effect of memory and hand-target on proprioceptive localization. Neuropsychologia. 2012;50: 1462-1470.

doi:10.1016/j.neuropsychologia.2012.02.031

63. Reuschel J, Rösler F, Henriques DYP, Fiehler K. Spatial updating depends on gaze direction even after loss of vision. J Neurosci. 2012;32: 2422-2429. doi:10.1523/JNEUROSCI.2714-11.2012

64. Goble DJ, Coxon JP, Wenderoth N, Van Impe A, Swinnen SP. Proprioceptive sensibility in the elderly: Degeneration, functional consequences and plastic-adaptive processes. Neuroscience and Biobehavioral Reviews. Pergamon; 2009. pp. 271-278. doi:10.1016/j.neubiorev.2008.08.012

65. 't Hart BM, Ayala MN, Henriques DYP. Proprioception and prediction do not optimally integrate in hand localization. San Diego: Society for Neuroscience; 2018. Available:

https://www.abstractsonline.com/pp8/\#!/4649/presentation/11269 